\title{
Kernos
}

Revue internationale et pluridisciplinaire de religion

grecque antique

15 | 2002

Varia

\section{La « logique » du récit mythique dans l'ode rhodienne de Pindare (Pind. O. VII)}

\section{María Carmen Barrígon Fuentes}

\section{OpenEdition}

Journals

Édition électronique

URL : http://journals.openedition.org/kernos/1362

DOI : 10.4000/kernos.1362

ISSN : 2034-7871

Éditeur

Centre international d'étude de la religion grecque antique

Édition imprimée

Date de publication : 1 janvier 2002

ISSN : 0776-3824

Référence électronique

María Carmen Barrígon Fuentes, «La « logique » du récit mythique dans l'ode rhodienne de Pindare (Pind. O. VII) », Kernos [En ligne], 15 | 2002, mis en ligne le 21 avril 2011, consulté le 05 mai 2019. URL: http://journals.openedition.org/kernos/1362; DOI : 10.4000/kernos.1362 


\section{La " logique " du récit mythique dans l'ode rhodienne de Pindare (Pind. O. VIII) ${ }^{1}$}

Pindare, pour célébrer la victoire olympique du pugiliste Diagoras de Rhodes, de la famille des Ératides, composa en l'an 464 av. J.-C. la septième Olympique, l'une des odes les plus célèbres et qui a suscité de nombreuses interprétations ${ }^{2}$.

Il faut remarquer que les travaux les plus récents sur cette épinicie se concentrent surtout sur l'appréciation du mythe, même si, au moment de l'aborder, elles le font selon des perspectives différentes. D'un côté, certains auteurs ont essayé de trouver dans cette ode une «Grundgedanke $»^{3}$. Par exemple, des auteurs comme G. Norwood ${ }^{4}$ et $G$. Lawall ${ }^{5}$ voient un noyau unique prédominant dans l'ode et l'identifient grâce à l'homophonie entre le nom de l'île Rbodos et de la rose rbodon, avec le processus de naissance et de croissance de la fleur. En revanche, d'autres auteurs tentent d'interpréter le mythe en fonction du complexe d'idées exprimées dans l'ensemble de la composition poétique ${ }^{6}$, certains tiennent compte des idées morales et religieuses du poète, tel que J. Defradas ${ }^{7}$, ou des problèmes de type politicosocial et économique évidents dans la société de son temps, comme

1 Cette étude a été réalisée dans le cadre du Programme de Recherche PB-97-0403 de la DGICYT espagnole.

2 Selon un ancien témoignage rapporté par les scholies (Drachmann I 195), elle fut gravée en lettres d'or dans le temple d'Athéna, à Lindos (GoRgoN, FGrHist 515 F 18).

3 Cette théorie, défendue en particulier par F. Mezger (Pindars Siegeslieder, Leipzig, 1880) et Young (1968, p. 69-105), mène, selon VERDENIUs et BRAswell, à une dangereuse hyperexégèse. L'interprétation de Young résulte d'une extension des idées suggérées par Lawall ( $c f$. note 4), comme lui-même l'admet. $C f$. l'orientation bibliographique, bien résumée par D.E. Gerber, "Studies in Greek Lyric Poetry: 1967-1975", CW 70.2 (1976), p. 142-143; "Studies in Greek Lyric Poetry: 1975-1985", CW 81 (1988), p. 454-457; "Pindar and Bacchylides 1934-1987", Lustrum 31 (1989), p. 202-207. Verdenius réplique aux commentaires de BArkhuizen (1968), p. 25-37; Braswell. (1976), p. 233-242 et Defradas (1974), p. $34-50$.

4 Pindar, Berkeley, 1945, p. 138-145. Repris partiellement par LAwALl (1961, p. 33-47), il rapelle que la rose figurait sur les monnaies rhodiennes et pour lui, l'île n'émerge pas, mais « fleurit».

5 Cf. (1961), p. 33-47.

6 Cf. SMITH (1969), p. 172-175; RuBin (1980).

7 (1974), p. 34-50 
A. Bresson ${ }^{8}$. D'autres, par contre, l'étudient à partir des schémas structuraux sur lesquels chaque mythe serait construit; tel est le cas de J.H. Barkhuizen ${ }^{9}$, J.T. Hooker ${ }^{10}$ ou C. Dougherty ${ }^{11}$; ou en analysant la fonctionnalité des récits mythiques en fonction de l'occasion, comme P.A. Bernardini ${ }^{12}$.

Nous nous proposons d'attirer l'attention sur l'organisation interne particulière de la VII Olympique de Pindare, à travers laquelle le poète aura recours à toute sa capacité créative pour transmettre un message plus efficace que celui d'un simple récit. Dans ce but, il utilise un code mythique partagé par la société à laquelle il s'adresse, et il établit un rapport étroit entre le récit mythique, l'actualité et la gnomè. Il réalise une structuration dans laquelle sa narration s'écarte du récit linéaire historique en utilisant une technique " progressive » dans le récit mais « régressive » dans la chronologie réelle, c'est-à-dire qu'il intègre dans le déroulement du poème une manipulation constante de l'axe du temps, comme l'a fait remarquer A. Hurst ${ }^{13}$.

Ce poème se compose de cinq triades et il est conçu comme un cercle qui s'ouvre avec la présentation de l'athlète Diagoras, dont il souligne la puissance physique, les couronnes, la spécialité sportive, de qui il est le fils et l'origine de sa famille, et qui se ferme en retournant à nouveau à la figure du vainqueur avec l'énumération de ses multiples triomphes. La partie centrale est occupée par un triple mythe développé entre les vers 20 et 76, comprenant la seconde, la troisième et la quatrième triade.

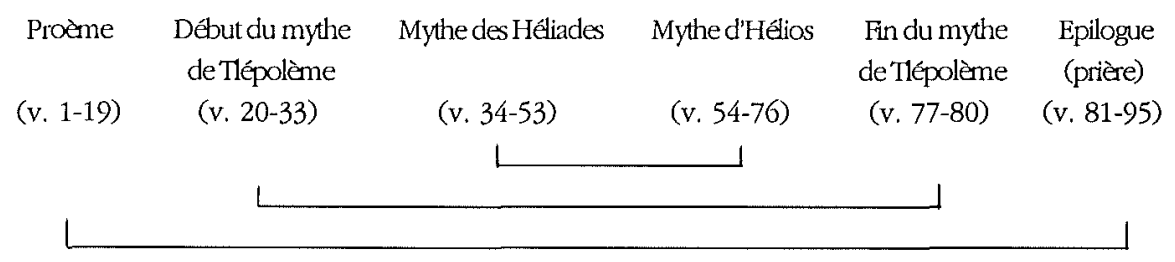

Les trois légendes racontées représentent des moments d'une seule fresque, où le fil conducteur est le passé légendaire de l'île de Rhodes. Du point de vue structural, on aperçoit une disposition en cercles concentriques qui encadrent des mouvements de régression-retour. Le premier mythe est le plus récent dans l'histoire de Rhodes et il se réfère à Tlépolème et à sa colonisation de l'île (v. 20-33); le second rapporte la naissance d'Athéna et l'implantation de son culte par les Rhodiens suivant les instructions d'Hélios (v. 34-53); et le troisième, le plus ancien, expose la naissance de Rhodes et le

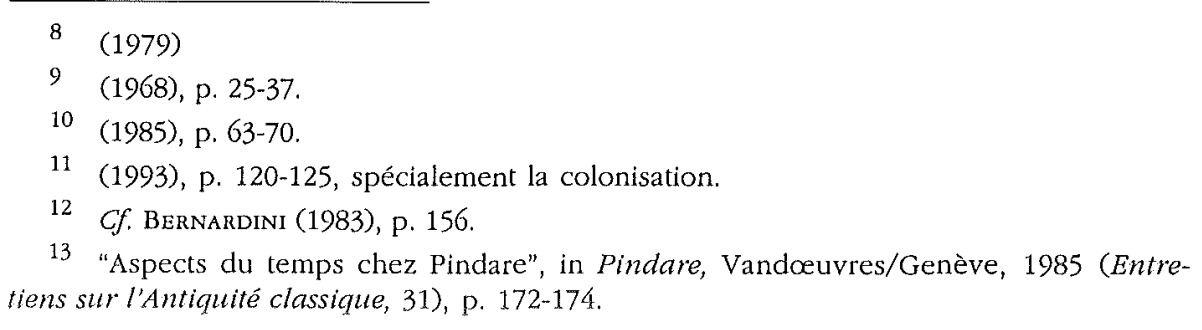


mariage d'Hélios avec la nymphe éponyme de l'île (v. 54-76) ${ }^{14}$. Les trois mythes ne sont pas racontés en détail, mais Pindare les synthétise en quelques lignes, qui constituent le $\kappa \in \phi a ́ \lambda$ aıov d'une structure en anneau ${ }^{15}$.

La structure interne des trois mythes est similaire et tourne autour de deux éléments essentiels : une erreur ou un oubli et l'intervention favorable des dieux (observés très souvent par les commentateurs), c'est-à-dire, le récit des faits est construit sur le schéma : faute/privilège divin. Dans chacun des mythes, le poète interrompt la narration avec un ou deux passages gnomiques. Effectivement, l'action de chaque mythe est déchaînée par une áfaptía: la première est le meurtre de Licymnios par Tlépolème qui se laisse emporter par la colère; la seconde est l'oubli du feu pour le sacrifice à Athéna de la part des Rhodiens, et la troisième est la marginalisation d'Hélios lors de la partition du monde. Mais, paradoxalement, loin d'entraîner une conséquence néfaste, chacune de ces fautes devient un privilège divin pour l'île et ses habitants : de la transgression de Tlépolème naît le peuple dorien de l'île de Rhodes, de l'oubli du feu, son prestige intellectuel et artistique, et de la marginalisation d'Hélios, le patronage du Soleil sur l'île.

Voyons le mécanisme de chaque mythe. L'ode commence par un proème qui développe l'image d'un banquet de noces dont se sert le poète pour offrir à Diagoras son chant, telle une coupe d'or débordant du nectar de sa poésie (v. 1-12). Cette image crée un réseau de motifs qui constitue le fondement de l'éloge, qui a été analysé en profondeur par plusieurs auteurs ${ }^{16}$. La correspondance de la charis du symposium se trouve à la fin de l'ode dans l'expression

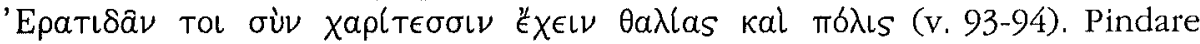
commence la strophe, l'antistrophe et l'épode de cette première triade avec un mot clef : Tıs - $\dot{\epsilon} \gamma \dot{\omega}$ - kal $\nu v \nu$ respectivement, en établissant une parfaite contraposition. Avec kal vuv, il initie le moment d'actualité pour présenter Diagoras et nommer pour la première fois l'île, fille d'Aphrodite et épouse d'Hélios ${ }^{17}$.

\section{Le mythe de Tlépolème}

Dans la seconde triade, occupant la strophe, l'antistrophe et une partie d'épode, le poète aborde le premier des épisodes mythiques dans le triptyque dédié à l'histoire légendaire de Rhodes, la légende de Tlépolème d'Argos

14 Pour les citations du texte grec, je suis l'édition de B. SNell, H. Maehler, Pindamis. I. Epinicia, Leipzig, $1980^{6}$, et pour la traduction du texte, celle de A. Puech, Pindare, vol. I, Olympiques, Paris, Les Belles Lettres, 1949, p. 87-100.

15 L. ILLIG, Zur Form der pindariscben Erzäblung. Interpretationen und Untersucbungen, Leipzig, 1931, p. 57.

16 Cf. Braswell (1976), 233-242; Bernardini (1983), p. 157-163; Brown (1984), p. 37-50.

17 Dans le mythe traditionnel, la mère de Rhodes est Amphitrite ou Halia. Cf. Drod. Sic., V, 55, 2; Apollod, Bibl. II, 4, 5. On croit que la généalogie pindarique fut inventée pour faire ressortir la beauté de Rhodes. Cf. U. von Wramovirz, Pindaros, Berlin, 1922, p. 364; Young (1968), p. 76. 
(v. 20-33). Le mythos - qui, pour Pindare, ne désigne que le mensonge devient logos qui garantit la vérité et qui lie l'actualité de la victoire au passé " mythique » des ancêtres du vainqueur et des origines de l'île, en ayant recours aux aitia des principales traditions religieuses des Rhodiens.

Les événements racontés appartiennent au passé relativement récent de 1'île car il s'agit de sa colonisation par le héros, fils de Héraclès ${ }^{18}$ et d'Astydamie $^{19}$ dans la version pindarique - par conséquent, descendant de Zeus -, qui pour expier l'assassinat de son oncle maternel Licymnios, après avoir consulté l'oracle de Delphes, arrive sur l'île tel un exilé.

Les raisons générales du choix d'un tel mythe sont faciles à comprendre : d'une part, honorer la patrie du vainqueur en réveillant le souvenir d'événements illustres, en associant la colonisation de toute l'île aux Argiens et, en dernier lieu, à Héraclès, et puis par ailleurs louer les Ératides, le genos qui descendait le plus directement de Tlépolème avant de servir de propagande pour les projets politiques de cette famille ${ }^{20}$.

Cette version pindarique semble une modification intentionnelle de celle que nous trouvons dans le "Catalogue des Vaisseaux » de l'Iliade (II, 653670), selon l'annonce du poète lui-même, lorsqu'il dit qu'il souhaite à travers son message opérer une rectification de l'histoire commune ( $\xi u \nu o ̀ \nu ~ a ́ \gamma \gamma \hat{\epsilon} \lambda \omega \nu$

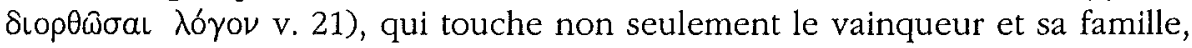
mais aussi la ville et ses habitants. Effectivement, il y a des différences : tandis qu'Homère omet le motif du crime et que la tradition ultérieure parle de meurtre involontaire ${ }^{21}$, Pindare le décrit comme un acte de colère ( $\chi 0 \lambda \omega \theta \in l_{S} \mathrm{v}$. 30); face à l'idée homérique de maintenir volontairement Tlépolème en exil errant partout ${ }^{22}$, ici le navigateur va droit au but, l'île de Rhodes, afin de

18 Prinz (1979), p. 78-97. Pour la généalogie de Tlépolème, $c f$. Apollod., II, 49, 52.

19 Les sources littéraires transmettent différents noms pour la mère de Tlépolème : selon l'rliade (II, 658), elle s'appelle Astyoché ou Astyocheia (cf. Apollod, epitome III, 13; Hygin, fab. 97, 7), Astydamie selon Pindare (O. VII, 24) et Hésiode (fr, 232 Merkelbach/ West), mais selon PhÉRÉcyde (FGrH 3 F 80 Jacoby), Astygéneia (cf. A kousilaos, FGrH 2 F 44 Jacoby).

20 Cf. Pavese (1975), p. 100 et Bresson (1979), p. 150-151. Selon Pindare la séquence est $:$ Zeus $\rightarrow$ Héraclès $\rightarrow$ Tlépolème $\rightarrow$ Ératides ? $\rightarrow$ Callianax $\rightarrow$ Damagètos $\rightarrow$ Diagoras.

21 Diod. Sic., IV, 58, 7-8; V, 59, 5-6; Apollod., Bibl. II, 8, 2 et Schọl. Pind. O. VII, 36, p. 208-209 Dr. Cf. Strabon, XIV, 2, 6 et Nonnos, Dion. XIV, 41.

22 Dans les poèmes homériques, comme L. Gil lui-même l'écrit : « el exilio fue la solución de compromiso que se arbitró primero en los delitos de sangre habidos en el seno de una familia, donde los lazos de consaguineidad y alianza dificultaban la aplicación estricta de la pena del Talión », Introducción a Homero, II, Barcelona, 1984, p. 389. Comparer aux paroles de Théoclymène dans l'Odyssée (XV, 272-278). Les exemples sont nombreux dans la poèsie épique. $C f$. M. Gagarin, Drakon and Early Atbenian Homicide Law, New Haven/London, 1981, p. 6-18 et R. PARKer, Miasma. Pollution and Purification in Early Greek Religion, Oxford, 1983, p. 130-136, pour le débat sur le meurtre et sa punition dans les poèmes homériques, dans lesquels il n'y a pas le concept de la

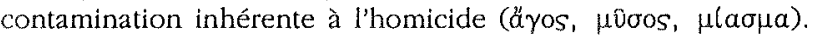


réaliser la parole de l'oracle, tel un $\chi \theta 0 \nu$ òs olkıorń $\rho^{23}$. Pindare fait confluer, d'un côté, l'expédition de colonisation, conséquence d'une réponse oraculaire correspondant à une activité réelle du sanctuaire delphique en tant que promoteur de ce type d'actions ${ }^{24}$, et, de l'autre, la nature rituelle de l'expiation du crime de sang exigeant l'expulsion hors de la communauté de l'individu souillé par cet acte. La transformation de Tlépolème en héros civique n'est possible qu'à travers la purification. Or c'est le rôle que joue l'oracle de Delphes; en conséquence, son expédition représente la purification nécessaire pour expier le crime ${ }^{25}$, et précisément cette réinterprétation du mythe permet à Pindare d'établir une comparaison entre Diagoras et

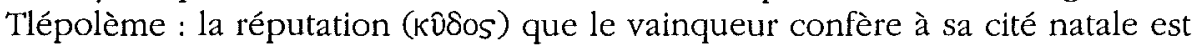
renforcée dans le poème par la figure de Tlépolème, un assassin transformé

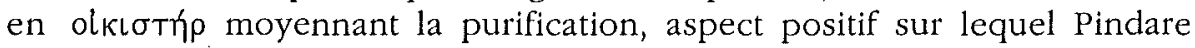
insiste avec ténacité. Malgré tout, le schéma narratif que Pindare nous offre: situation critique $\rightarrow$ consultation et intervention de l'oracle d'Apollon $\rightarrow$ exclusion de la communauté moyennant la colonisation, est commun à d'autres récits, qui soulignent les aspects religieux et la participation d'Apollon dans les situations critiques, car son intervention permet au héros de commencer une nouvelle vie sous le signe de la purification ${ }^{26}$.

La valeur de $\chi 0 \lambda \omega \theta \in$ is semble renforcée par le passage gnomique qu'utilise

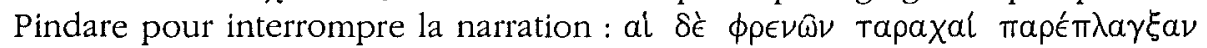
kal бoфóv (« le trouble de l'esprit égare même le sage »v. 30-31); et le langage particulier employé dans le vers 77 , lorsqu'il dit « reçoit la douce rançon de sa

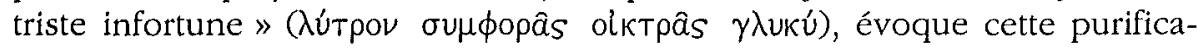
tion.

La première faute dans ce mythe est donc la mort par accident de Licymnios qui se résout de façon favorable avec la colonisation de l'île. Cette légende permet au poète d'attirer deux fois l'attention au travers de deux

23 Verdenius (1972), p. 57, ecrit que « in the original version of the story Rhodes was colonized not by Tlepolemus himself but by the third generation after him »; pour Prinz (1979), p. 96-97, il n'y a pas aucune réalité historique dernière ce récit mythique et il refuse la colonisation de Rhodes par Argos. $C f$. aussi E. Suárez de la Torre, "Gli oracoli relativi alla colonizzazione della Sicilia e delle Magna Grecia", QUCC 77 (1994), p. 7-37, spéc. p. 11.

24 Cf. M.P. Nizsson, Cults, Myths, Oracles and Politics in Ancient Greece, New York, 1951; H.W. Parke, D.E. Wormell, The Delpbic Oracle I, Oxford, 1956, p. 49-81; M. LOMBARDo, "La concezioni degli antichi sul ruolo degli oracoli nelli colonizzazione", in Ricerche sulla colonizzazione greca, Roma, 1972, p. 63 sq.; I. MalkiN, Religion and Colonization in Ancient Greece, Leiden, 1987.

25

26

Cf. Dougherty (1993), p. 120-130.

PARKE-WORMELL, o.c. I, p. 50-54 déjà avaient observé la répétition de cette séquence dans les mythes, mais non dans les récits historiques. Cf. MALkın, o.c., p. 41-43 et Suárez de la Torre, l.c., p. 11 sq. Dougherty ne considère pas le meurtre de Licymnios comme une faute dans la mesure où Pindare en fait l'exagération mythique d'un sport violent (1993, p. 126). 
gnomai sur le motif des fautes ( $\alpha \mu \pi \lambda \alpha k l a\llcorner v .24)$ ou des bouleversements de l'âme ( Tapaxaí v. 30), entre lesquelles s'inscrit le récit du crime commis par le héros : la première anticipe la faute et la seconde sert de justification, en reconnaissant l'instabilité de la vie de l'homme, poursuivi par de multiples erreurs, même si celles-ci peuvent se résoudre de façon positive grâce à la présence divine ${ }^{27}$.

Dans ce première récit mythique qui commence au vingtième vers, mais qui n'aboutit qu'au vers 77 , s'enchaînent les deux autres excursus sur la légende de Rhodes qui représentent de clairs témoignages des faveurs des dieux à l'île et à ses habitants. À partir du vers 33 s'interrompt le thème de Tlépolème pour permettre au poète de remonter en arrière au niveau mythique avec des narrations de type étiologique sur les cultes et les caractéristiques de l'île, bien évidemment préalables à la colonisation dorienne que représente Tlépolème, mentionné à nouveau au vers 77 .

\section{Le mythe des Héliades}

Entre les vers 34 et 53 s'inscrit le second mythe, celui des Héliades, occupant la fin de l'épode de la seconde triade jusqu'à la première partie de l'épode de la troisième triade.

Le texte se déroule comme un récit circulaire qui commence par la pluie d'or envoyée par Zeus et la naissance d'Athéna de la tête de Zeus, grâce à l'aide et à l'habileté d'Héphaistos ${ }^{28}$, et s'achève par la pluie d'or que Zeus envoie, une fois institués les sacrifices en l'honneur d'Athéna, qui leur octroie la techne $\ddot{29}^{29}$. La naissance d'Athéna sert ici à préparer et, dans une certaine mesure, à comparer une autre naissance prodigieuse, celle de Rhodes, émergée des eaux de la mer dans le mythe suivant. Ici, Rhodes est déjà promise à Hélios, qui prescrit à ses fils aimés, les Héliades, le premier culte à Athéna en élevant un autel et en y instituant un pieux sacrifice fumant

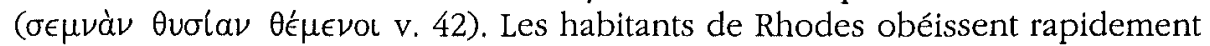
à l'ordre d'Hélios, mais dans leur hâte, ils oublient le feu prévu pour le sacrifice et ils se voient obligés de réaliser des sacrifices apyra.

Ceci est le point central qui illustre le choix et la fonction du second mythe. De la même façon, la stratégie narrative consiste à organiser le récit

27 Voir les intéressantes études de Sullivan (1982), p. 217-223 et Theunissen (2000), p.79-100. Pour la discussion sur l'interprétation de amplakiai, cf. J.M. BREMER, Hamartia, Amsterdam, 1969, p. 112-117; SmIth (1969), p. 176-177; VERdenius (1972), p. 58-59; Defradas (1974), p. $40 \mathrm{sq}$. Pour le concept de l'instabilité de la vie, cf. Simonide, PMG 521 Page.

28 Pindare omet les motifs de cette naissance prodigieuse. Cf. RuBIN (1980), p. 67-79.

29 Il y a différentes interprétations pour la pluie d'or. Young (1968), p. 84 sq. parlait de deux pluies. Pour Verdenius (1972, p. 21), c'était aussi le cas. Cependant, revenant sur le problème (1976, p. 243-253), il accueillait l'objection de Willcok (1975), p. 6, en acceptant la possibilité d'une seule pluie, contestée par L. Lennus, Pindaro. Olimpiche, Milano, 1981, p. 122. Cf. CANtilena (1987), p. 209-216. Sur l'alternance de l'image de «neige » et de « pluie», cf. Young (1968), p. 84 sq. 
pour qu'à la fin y apparaissent les privilèges divins envers les Rhodiens et à interrompre la narration au moyen d'une gnomè avant d'exposer la faute commise. Tout comme, dans l'épisode mythique antérieur, les erreurs atteignaient la volonté de l'homme, ce sera maintenant l'image du "nuage de

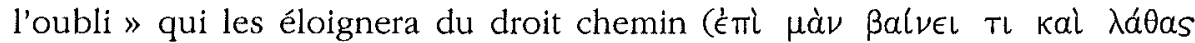

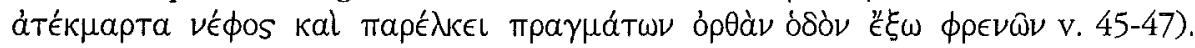
La faute est moins grave que celle commise par Tlépolème, même si elle reste scandaleuse et hors normes. Les dieux, comme ils l'avaient fait auparavant avec Tlépolème, pardonnent cette nouvelle erreur et, au lieu d'attirer le châtiment divin, Zeus fait tomber sur Rhodes une "pluie d'or » et Athéna leur confère le domaine des arts, c'est-à-dire la technè qui leur permettra d'élaborer d'habiles artifices ${ }^{30}$ en leur démontrant de cette manière qu'elle a accepté la coutume de sacrifier sans feu.

Cet aspect a été analysé par P. Sfyroeras ${ }^{31}$ et I. Chirassi Colombo ${ }^{32}$. Le premier soutient que l'origine des sacrifices sans feu à Rhodes doit être analysée en contraste avec l'aition de la course de torches des Panathénées athéniennes et il propose que les raisons du choix de ce mythe sont, d'un côté, les circonstances historiques $c a .464$ av. J.-C. et l'intérêt de l'aristocratie de l'île à renforcer ces origines doriennes; et, de l'autre, la tendance au panhellénisme de la poésie pindarique. Nous partageons l'opinion de Suárez de la Torre ${ }^{33}$ qui souligne que « le problème principal est celui du poids que la vision panhellénique peut avoir dans une ode interprétée à Rhodes pour une famille rhodienne : il faut en tout cas supposer une bonne connaissance des traditions athéniennes et un intérêt à les surpasser de la part des destinataires, avec un certain esprit de rivalité vis-à-vis des Athéniens ». Chirassi met en relief le fait que l'activité religieuse des Rhodiens n'a pas besoin de feu parce que la force du soleil était suffisante pour les sacrifices. Ses points d'appui sont les témoignages épigraphiques sur les apyroi bômoi. Bien que nous soyons devant une version purement locale avec une manipulation du mythe de la part de Pindare, le poète adapte la structure du poème au contraste faute/ récompense divine. Le nuage de l'oubli de la gnomè semble annoncer l'oubli du feu et la blonde nuée que Zeus envoie procure aux Héliades la richesse, mais elle leur apporte aussi la lumière. Pindare fait une synthèse nouvelle, originale, à laquelle une structure rigoureuse donne une signification globale, destinée à servir la cause des commanditaires de l'ode.

Nous voyons comment, au cours de la célébration de l'île, Pindare met en relief la primauté des Rhodiens due à leur richesse exubérante et à leur

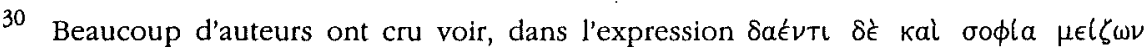
$\check{a} \delta 0 \lambda$ os $\tau \epsilon \lambda \epsilon \in \epsilon \imath$ (v. 53), une opposition entre cet art et la magie que la légende attribuait aux Telchines. Mais la sentence est générale et Pindare rationalise la tradition. $C f$. VERdeniUs (1972), p. 73; HOOKER (1985), p. 66; YOUNG (1987), 152-157.

31 (1993), p. 1-26

32 Lors de sa communication au colloque de Rhodes.

33 Cf. le compte rendu de cet article à paraitre dans la bibliographie Mentor. 
habileté technique sans égal, ce qui, selon Bernardini ${ }^{34}$ correspondrait sur le plan temporel à l'importance de l'artisanat et à l'opulence économique qui provient du commerce, notoires à cette époque-là. Ici, à nouveau, le poète modifie le modèle homérique où l'on fait allusion à la richesse, mais sans autres précisions ${ }^{35}$, et il augmente le motif de ce don divin indicatif du pardon. La conclusion de ces deux narrations mythiques anticipe et prépare le triomphe définitif de la nature représenté par le surgissement de l'île dans la dernière partie de la trilogie mythique, et en même temps il permet d'établir un parallèle avec Diagoras : étant donné que les Rhodiens ont obtenu des dieux la richesse, l'habileté et la célébrité, Diagoras obtiendra ainsi de Zeus le succès, la célébrité et le respect.

\section{Le mythe d'Hélios et de la naissance de Rhodes}

Le zénith est atteint avec le mythe de la naissance de Rhodes, troisième et dernière partie (v. 54-76), qui met fin à l'histoire de l'île. Dans le but de rendre son récit plus convaincant, Pindare a recours à d'anciennes traditions mythi-

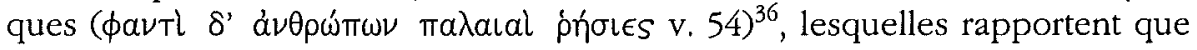
les dieux, en l'absence d'Hélios, oublièrent de lui octroyer sa part ( $\chi \omega$ úpas

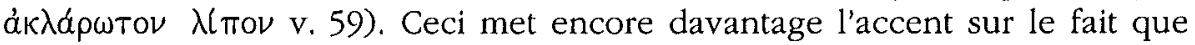

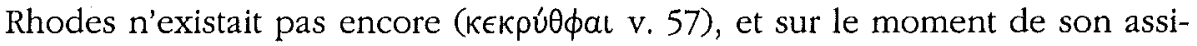
gnation postérieure à Hélios (v. 55-76). Zeus fut averti de l'erreur et fut sur le point de la corriger avec un nouveau partage, qui fut empêché par Hélios. Refus justifiable car ceci allait à l'encontre des lois grecques de l'anadasmos $^{37}$. Cependant Pindare le justifie d'une autre façon : parce qu'Hélios « voyait clairement comment du fond de la mer, surgissait une terre fertile pour les hommes et bénigne pour les troupeaux » (v, 60-64).

Une fois Rhodes née, Hélios s'unit à la nymphe éponyme de l'île, engendrant sept enfants, « auxquels il transmet, de tous les hommes des premiers âges, l'esprit le plus inventif » (v. 72-73). Nous voyons comment ces paroles s'apparentent à la tecbnè que leur confère Athéna (v. 50-52). De l'un d'entre eux - Cercaphos - vont naître les trois fondateurs des trois principales villes de l'île : Ialysos, Lindos, Camiros ${ }^{38}$. En rapport avec d'autres odes de Pindare, on reconnaît le modèle suivant pour le mythe choisi : union du dieu avec une

\footnotetext{
34 (1983), p. 171-178.

35 Il. II, 670.

36 Cf. Schol. PIND. O. VII, p. 222 Dr. qui assure qu'il n'existe pas de tradition historiographique sur les origines de Rhodes et que les poètes la connaissaient à travers d'anciens sages. Cf. K. FEHr, Die Mythen bei Pindar, Zürich, 1936, p. 132 sq., MüHLL (1963), p. 197-204; Young (1968), p. 87; Verdenius (1972), p. 74; Bernardini (1985), p. 181.

37 Cf. BREsson (1979), p. 19 sq; BerNaRdini (1983), p. 85-86.

38 Cf. Diod. Sic., V, 56; STRABON, XIV 2, 8. Ces trois cités rhodiennes correspondent à la division historique qui suit la colonisation dorienne, et la cité de Rhodes naît en 407 av. J.-C. du synécisme de ces trois cités. Cf. G. Pugliese Carratelli, "La formazione dello stato rodio", SCO 1 (1951), p. 80-85; M. MogG1, I sinecisme interstatali greci, Pisa, 1976.
} 
femme (île ou ville, ici, Hélios-Rhodes), garantie du serment et descendance des éponymes mythiques du territoire colonisé plus tard par les ancêtres du vainqueur ${ }^{39}$.

Par ce mythe, la famille de Diagoras, les Ératides, qui provenait d'Ialysos, ville dans laquelle le culte à Hélios était très ancien, ainsi que tous les citoyens de ce lieu devaient éprouver un sentiment de satisfaction en évoquant leur descendance directe de la divinité particulièrement vénérée et de la qualité d'Ialysos comme aîné des trois frères ${ }^{40}$.

Même si Verdenius ${ }^{41}$ pense que « regressive order is a characteristic of archaic narrative », dans ce cas, il possède un relief spécial. Le point de repère commun aux trois mythes, l'alternance erreur/privilège, se combine avec une technique de régression qui touche à tous les éléments des mythes, à travers un climax progressif : autant en ce qui concerne les auteurs de la faute que le type de transgression, on emploie la même technique de régression de la chronologie réelle : la première a été commise par un héros colonisateur, la suivante par les héros fondateurs et, en dernier lieu, par les dieux. Dans le premier cas, il s'agit d'un délit dans le domaine humain, un crime de sang; dans le second, c'est un oubli dans la sphère rituelle, en rapport avec la divinité, et, en dernier lieu, on décrit un oubli des dieux dans un temps primordial. En définitive, nous avons le schéma suivant:

\begin{tabular}{|lllll|}
\hline Tlépolème & $\rightarrow$ & Héliades & $\rightarrow$ & Dieu-même \\
\hline Meurtre & $\rightarrow$ & oubli & $\rightarrow$ & oubli dû à l'absence \\
\hline Colonisation & $\rightarrow$ & prosperité & $\rightarrow$ & l'ile-même \\
\hline
\end{tabular}

\begin{tabular}{|l|l|l|l|}
\hline & strophe & antistropbe & épode \\
\hline 2e triade & $\begin{array}{l}\text { ancêtres } \\
\text { Tlépolème }\end{array}$ & $\begin{array}{l}\text { meurtre } \\
\text { (transgression) }\end{array}$ & $\begin{array}{l}\text { oracle, colonisation } \\
\text { (récompense) }\end{array}$ \\
\hline 3e triade & culte à Athéna & $\begin{array}{l}\text { oubli du feu, } \\
\text { compensation Zeus }\end{array}$ & tecbnè d'Athéna \\
\hline 4e triade & oubli dû à l'absence & assignation de l'île & $\begin{array}{l}\text { union } \\
\text { Hélios-Rhodes }\end{array}$ \\
\hline
\end{tabular}

Le retour à l'actualité se fait à travers l'adverbe Tó Diagoras est utilisée par le poète dans le but de remonter à Tlépolème et la nouvelle mention est le point de retour du vainqueur puisqu'il a également

39 Cf. Suńrez de la Torre (1990), p. 354-355.

40 Cf. Morelli (1959), 96-102; A. Rivier, "Mythe et poésie. Leurs rapports et leur fonction dans trois épinicies de Pindare", in F. LAsserre, J. Sulliger (éds), Études de Littérature grecque, Genève, 1975, p. 259-288.

41 (1972), p. 61. 
obtenu des victoires aux jeux en son honneur et ce sont justement ces derniers qui permettront de faire le rapprochement entre le héros mythique et le héros actuel. Avec le vers 80, le poète ouvre l'éloge proprement dit de l'athlète, en énumérant la liste de ses victoires. Cette énumération n'a qu'une finalité informative, faire connaître à l'auditoire la carrière sportive du prestigieux pugiliste et de sa famille, mais elle a également une fonction eulogistique, dans la mesure où, plus elle sera importante, plus on mettra en valeur les mérites agonistiques du vainqueur.

Pindare conclut cette épinicie avec la prière à Zeus (v. 87-95), où le lexique utilisé concentre les idées exposées tout au long du poème :

1. L'invocation affecte l'athlète et sa famille, et se concentre sur le principe de continuité de la souche (v. 92-93). Zeus doit éviter que la lignée reste à nouveau occulte et ceci est possible parce qu'à une autre époque, il

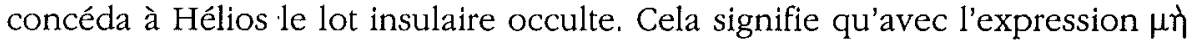

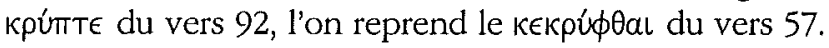

2. Les oublis du temps mythique vont être corrigés grâce à la navigation de Diagoras, bien qu'il doive être attentif aux vents. Le vainqueur suit un

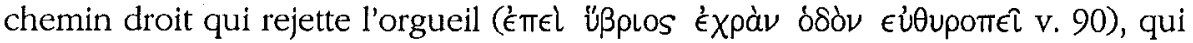
reprend l'idée du droit chemin du vers 46 . Le dernier contenu de la prière à Zeus concerne l'avenir de Diagoras et des Ératides, qui, comme celui de tous les hommes, doit subir des changements. En ce qui concerne l'instabilité et le côté provisoire de la vie de l'homme, le poète a déjà fait allusion à la fin du premier excursus mythique (v. 25-26), et il retourne dès lors une fois de plus à

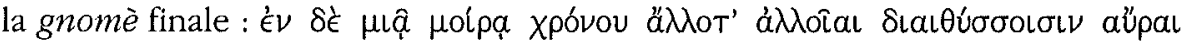
(v. 94-95). Diagoras et sa famille n'ont connu jusqu'à présent que le succès et la gloire, mais l'aide de Zeus leur est nécessaire en vertu de leur condition de epameroi. Les vents changent mais n'en sont pas pour autant un présage défavorable pour Diagoras. Il s'agit d'un appel à la faculté de jouir du moment présent tout en gardant à l'esprit l'incertitude d'un lendemain susceptible de changement.

3. Les principes de la sagesse héritée et de la descendance sont en rapport avec l'idée suggérée par l'image initiale de la coupe, tel un symbole de la transmission d'une famille à une autre, et en même temps expliquent et illustrent le senș de la phrase finale à propos du changement des vents. La

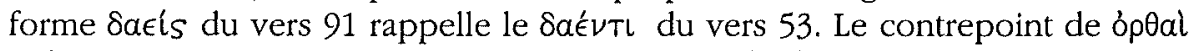

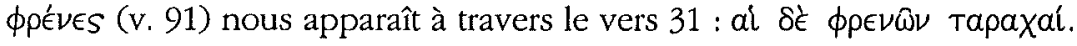

4. La défense de ses valeurs, en incluant également les valeurs agonistiques, trouvent leur contrepoint aux vers 5 et 11 .

5. L'aide divine que le poète lui-même a implorée, tel l'officiant d'un acte rituel, représente une garantie pour l'athlète qui connaîtra encore des succès, et pour le noble monsieur de Ialysos qui saura conserver la faveur de ses concitoyens. Le poète a prononcé une prière car la gloire et la célébrité de Diagoras ne connaissent pas d'ombre, et il a indiqué à travers le mythe que 
les dieux se montrent toujours favorables aux Rhodiens, de façon à ce que le commanditaire tout comme l'auditoire soient satisfaits.

Nous pouvons conclure en constatant que Pindare, pour procéder à la louange de Diagoras, le fait apparaître explicitement sur le fond des légendes relatives à la communauté qui est la sienne. La victoire de Diagoras glorifie Rhodes, et Rhodes, en retour, par la profondeur des plans chronologiques qu'elle offre, permet de voir la carrière de Diagoras comme une manifestation supplémentaire de la faveur divine. La disposition en cercles concentriques qui encadrent des mouvements de régression-retour favorise l'évolution de l'ode vers un climax représenté par la naissance de l'île de Rhodes et l'éloge proprement dit de l'athlète. Pindare, en altérant l'ordre des mythes, fait confluer les Héliades et les Ératides, qui possèdent des vertus semblables. Du point de vue conceptuel, les termes les plus remarquables sont $\chi a ́ p ı s, \lambda o ́ \gamma o s$, $\phi \rho \eta ̂ \nu, \sigma o \phi l a$, et la réitération du passage du négatif au positif ou, si l'on veut, de l'obscurité à la lumière, ou de la faute au bonheur.

Carmen BARRIGóN

Universidad de Valladolid

Espagne

\section{Orientation Bibliographique}

J.H. Barkhurzen, "Structural Patterns in Pindar's Seventh Olympian Ode", AClass 11 (1968), p. 25-37.

-, "Pindar's Seventh Olympian Ode: Comments on Verdenius' Commentary", AClass 23 (1980), p. 107-110.

P.A. Bernardini, "Le Halieia di Rodi e lo scolio a Pind. 0. 7,14 ab (p.229 Dr.)", Stadion 3 (1977), p. 1-3.

-, Mito e attualità nelle odi di Pindaro, Roma, Ed. dell'Ateneo, 1983, p. 155-192.

B.K. Braswell, "Notes on the Prooemium to Pindar's Seventh Olympian Ode", Mnemosyne 29 (1976), p. 233-242.

A. Bresson, Mythe et contradiction. Analyse de la vif Olympique de Pindare, Paris, 1979.

-, "Deux légendes rhodiennes", in Les grandes figures religieuses. Fonctionnement pratique et symbolique dans l'antiquité (Besançon 25-26 auril 1984), Paris, 1986, p. 411-421.

B. Brown, "The Bridegroom and the Athlete: The Proem to Pindar's Seventh Olympian, in D.E. Gerber (éd.), Greek Poetry and Pbilosopby: Studies in Honour of L. Woodbury, Chico, 1984, p. 37-50.

M. Cantilena, "Due studi sulla VII Olimpica di Pindaro, I: Quando cadde la pioggia d'oro?", Prometheus 13 (1987), p. 209-216.

M.T. Clavo, "El olvido en la Olímpica VII", Ittaca I (1985), p. 57-78.

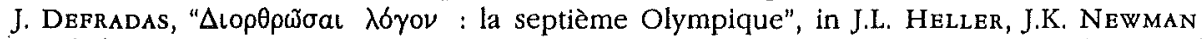
(éds), Serta Turyniana. Studies in Greek Literature and Paleograpby in bonor of Alexander Turyn, Urbana-Chicago-London, 1974, p. 34-50.

R. Dos Santos, "A sétima sinfonia de Píndaro", ELF 4 (1983/84), p. 25-43.

C. Dougherty, "Olympian 7 and Bacchylides Ode 1: Murder, Victory, and Colonization", in The Poetics of Colonization. From City to Text in Arcbaic Greece, Oxford, 1993, p. $120-135$.

J. Duchemin, "Pindare et l'Orient : le mythe de la VII ${ }^{e}$ Olympique. Essai d'interprétation", in Mélanges Edouard Delebecque, Aix-en-Provence, 1983, p. 109-130.

H. ERBSE, "Über Pindars Umgang mit dem Mythos", Hermes 127 (1999), p. 13-32. 
M.C. Fernández Llorens, "Comentarios estéticos de la séptima Olímpica", Helmantica 7 (1956), p. 357-377.

F. Ferrari, Olimpiche, intr., trad., e note, Milano, 1998.

H. von Geisau, "Tlepolemos. I", KlPauly V (1975), col. 877.

H. vaN GELDER, Geschichte der alten Rhodier, La Haye 1900, p. 19-27.

B. Gentili, "Riflessioni su mito e poesia nella Grecia antica", FAM 2 (1992), p. 7-22.

A. GostrolI, "Interpretazione metrica dell'Olimpica VII di Pindaro", AION(fil) 2-3 (1980/81), p. 59-66.

T.H. Hooker, "A Reading of the Seventh Olympian", BICS 32 (1985), p. 63-70.

L. KAHIL, "Tlepolemos", LIMC VIII, 1 (1997), p. 41.

A. KöнNкEN, Die Funktion des Mytbos bei Pindar. Interpretationen zu sechs Pindargedicbte, Berlin-New York, 1971.

G. Lawall, "The Cup, the Rose, and the Winds in Pindar's Seventh Olympian", RFIC 31 (1961), p. 33-47.

C. MORELLI, "I culti in Rodi", SCO 8 (1959), p. 69-70; 175-176.

-, I culti in Rodi, Pisa, 1959.

P. VON DER MUEHLL, "Weitere pindarische Notizien (Die rhodischen Sagen in der Siebenten Olympie)", $M H 20$ (1963), p. 197-204.

C.O. Pavese, "Le Olimpiche di Pindaro", QUCC 20 (1975), p. 65-121.

J. Poulloux, "Callianax, gendre de Diagoras de Rhodes", RPh 44 (1970), p. 206-215.

F. Prinz, Grïndungsmytben und Sagenchronologie, München 1979, p. 78-97.

F. Ribezzo, "Ad Pind. Ol. VII 43-44", RIGI9 (1925), p. 92.

N.F. Rubin "Pindar's Creation of Epinician Symbols: Olympian 7 and 6", CW 74 (1980), p. 67-87.

-, "Olympian 7: The Toast and the Future Prayer", Hermes 108 (1980), p. 248-252.

C.A.P. Ruck, "Marginalia Pindarica", Hermes 96 (1968), p. 129-142.

-, W.H. Matheson, "For Diagoras of Rhodes, Victory in boxing, Pindar, Olympian VII", Arion 4 (1965), p. 404-414.

J. Sснмірт, "Tlepolemos. I", in W.H. Roscher, Ausfübrlicbes Lexikon der griecbiscben und römiscben Mythologie, V, reimp. Hildesheim/New York, 1992, col. 1057-1061.

M. Segre, "L'oracolo di Apollo Pythaeus a Rodi", PP 4 (1949), p. 72-82.

—, "Rituali rodii di sacrifici", PP 6 (1951), p. 139-153.

P. Sfyroeras, "Firelees sacrifices: Pindar Olympian 7 and the. Panathenaic festival", $A J P b$ 114 (1993), p. 1-26.

O. SmITH, "An Interpretation of Pindar's Seventh Olympian Ode", Class. Med. 28 (1969), p. 172-175.

E. SuÁREz DE LA TORRE, "Parole de poète, parole de prophète : les oracles et la mantique chez Pindare", Kernos 3 (1990), p. 354-355.

S.D. Sullivan, "A Strand of Thought in Pindar, Olimpians 7", TAPbA 112 (1982), p. 215-223.

M. Theunissen, Pindar. Menschenlos und Wende der Zeit, Mannheim, 2000.

W.J. Verdenius, Pindar's Seventh Olympian Ode. A Commentary, Amsterdam/London, 1972

-. "Pindar's Seventh Olympian Ode. Supplementary Comments", Mnemosyne 29 (1976), p. 243-253.

W.J. Vian, "C.R. de l'ouvrage de W.J. Verdenius, Pindar's Seventb Olympian Ode. A Commentary", RPb 48 (1974), p. 334-335.

U. von Wilamowitz-MoellendorfF, Pindaros, Berlin, 1922, p. 360-368.

M.M. WILlcock, "Review: W.J. Verdenius: Pindar's Seventh Olympian Ode. A Commentary", CR 25 (1975), p. 5-6.

E. WüST, "Tlepolemos. I", $R E$ VI (1937), cols. 1614-1618.

D.C. Young, Three Odes of Pindar. A Literary Study of Pythian 11, Pythian 13 and Olympian 7, Leiden, 1968 (Mnemosyne, Suppl. 9), p. 69-105.

-, "Pindar and Horace Against the Telchines (Ol. 7.53 \& Carm. 4.4.33)", AJPb 108 (1987), p. $152-157$. 